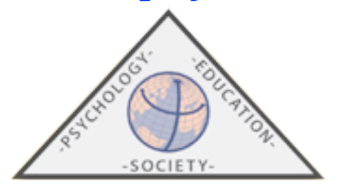

\title{
Una perspectiva australiana sobre el desarrollo profesional docente en tiempos supercomplejos
}

\author{
Lorraine M. LING* ${ }^{*}$ \& Noella M. MACKENZIE** \\ (*) La Trobe University, $\left({ }^{* *}\right)$ Charles Sturt University (Australia)
}

(Recibido el 20 de febrero de 2015; Aceptado el 27 de Julio de 2015)

\begin{abstract}
RESUMEN: Desarrollo Profesional Docente es una de los muchos términos utilizados para referirse a la educación y formación de los docentes en ejercicio. En este artículo, los autores abordan los tipos de desarrollo profesional que se ofrecen actualmente en Australia y los comparan con los tipos de desarrollo profesional que los profesores pueden necesitar para desempeñarse en la era de la supercomplejidad, donde hay incertidumbre, inseguridad $\mathrm{y}$ un futuro desconocido e incierto. El desarrollo profesional es definido como un proceso que implica múltiples agentes. También se analiza críticamente la influencia de los gobiernos y de otros organismos externos.
\end{abstract}

Palabras clave: profesores; aprendizaje profesional; resultados del aprendizaje; futuros desconocidos; incertidumbre.

\section{An Australian perspective on teacher professional development in supercomplex times}

\begin{abstract}
Professional Development is one of many terms given to the in-service education and training of teachers. In this paper, the authors address the kinds of professional development currently offered in Australia and compare it with the kinds of professional development teachers may require to deal with an era of supercomplexity, where there is uncertainty, insecurity and an unknown and unknowable future. Professional development is seen as involving multiple stakeholders and the influence of governments and other external bodies is also critically examined.
\end{abstract}

Keywords: teachers; professional learning; learning outcomes; unknown futures; uncertainty.

Correspondencia: Emeritus Professor Lorraine M. Ling. La Trobe University. Bundoora 3086. Victoria, Australia. Email: L.Ling@1atrobe.edu.au 


\section{Cómo citar este artículo}

Ling, L. M., \& Mackenzie, N. M. (2015). Una perspectiva australiana sobre el desarrollo profesional docente en tiempos supercomplejos (trad. al castellano de A. González Royo). Psychology, Society and Education, 7(3), 370-386. [V.O.: An Australian perspective on teacher professional development in supercomplex times. Psychology, Society and Education, 7(3), 263-278].

\section{Introducción: Futuros Desconocidos}

La educación del siglo 21 es complicada debido a nuestro futuro desconocido (Barnett, 2012). Al afirmar que el futuro es desconocido nos referimos a una situación en la que el conocimiento es siempre revisable y provisional, donde las habilidades necesarias para el futuro son desconocidas, dado que los tipos de contextos en los que vamos a vivir y trabajar son en gran parte inciertos. En buena medida, el futuro es siempre desconocido. Sin embargo, en una época de supercomplejidad, esta situación se ve agravada por el hecho de que los marcos y reglas con los que se evalúa el contexto actual y el posible contexto futuro están en constante evolución y cambio. Mientras que algunos pueden afirmar que el futuro ha sido siempre desconocido, en la época contemporánea se produce "un nuevo desafío pedagógico, si no urgencia" [“a new pedagogical challenge if not urgency”] (Barnett, 2012, p. 65). El impacto de los futuros desconocidos en la enseñanza y el aprendizaje es significativo y redefine especialmente la forma en que los profesores y los alumnos conciben el conocimiento y las habilidades.

El Desarrollo Profesional Docente (DPD) necesario para preparar a los profesores de forma que guíen a sus alumnos hacia un futuro desconocido necesita aportar conceptos e ideas que dirija a los profesores hacia nuevas maneras de entender su trabajo como experimentadores, exploradores, innovadores y profesionales arriesgados. Muchos de los programas actuales de DPD en Australia, especialmente aquellos diseñados para entrenar a los docentes en programas específicos o para el uso de determinados materiales ya publicados, son dogmáticos y prescriptivos, y dejan poca o ninguna opción para que el profesor interactúe creativamente con el currículo o con nuevos enfoques pedagógicos. Es poco probable que tales programas puedan preparar a los profesores o a los alumnos para enfrentarse a lo desconocido. Nuestro futuro incierto nos obliga a dar un "giro ontológico" (Barnett, 2012, p. 65), que nos impulsa a repensar qué es ser un profesor y ser un aprendiz, dado que estas identidades están cambiando al igual que cambian nuestros marcos de referencia previos. Los programas de DPD tienen la capacidad de conducir y facilitar tales replanteamientos, como se expone en este artículo.

\section{EI DPD en Australia Actualmente: ¿Cómo Es?}

Muchas términos han sido utilizados para referirse al DPD: aprendizaje profesional, desarrollo profesional continuo, educación en-servicio y formación en el lugar de trabajo son sólo algunos ejemplos. El DPD generalmente se refiere a las oportunidades de formación ofrecidas a los profesores en ejercicio y a los formadores 
de docentes. En muchos casos, el DPD se inicia y es ofrecido por los propios profesores, aunque en el contexto australiano actual es también ofrecido con frecuencia por asociaciones profesionales, editoriales y consultores privados, con las universidades entrando cada vez de forma mas seria en este terreno durante las últimas dos décadas. Asimismo, el DPD en Australia se ofrece cada vez más a través de seminarios on-line o de talleres en línea y cursos cortos.

Como antecedente, puntualizamos aquí que la formación inicial docente mínima en Australia es de cuatro años de duración. Puede componerse o bien de un título de grado (licenciatura) tras el bachillerato, o bien de un curso de posgrado de dos años de duración tras una diplomatura o licenciatura general en disciplina, de tres o cuatro años de duración. Como formadores de docentes en Australia, nosotros nos esforzamos en enfatizar que no puede y no debe esperarse que los programas iniciales de formación del profesorado produzcan profesores expertos, preparados para y capaces de hacer frente de inmediato a todas las complejidades de la enseñanza. Se necesita tiempo, experiencia y un aprendizaje permanente. Por lo tanto, es fundamental para los profesores continuar el DPD a lo largo de su carrera, siendo una expectativa y a menudo un requisito, como en cualquier otra profesión. En este artículo utilizamos la expresión "lugares de trabajo educativos" refiriéndonos a todas las posibles ubicaciones de la enseñanza, como por ejemplo escuelas, centros de educación infantil, colegios y universidades.

El Instituto Australiano para la Enseñanza y Liderazgo Escolar (acrónimo en inglés, AITSL) es el órgano regulador y de acreditación general para los programas de formación del profesorado en Australia. Este Instituto realizó un estudio en 2011 para conformar un marco de DPD nacional para profesores y directores escolares en Australia. En ese estudio, se identificaron cuatro principios generales para un aprendizaje profesional de calidad y eficaz. Los principios identificados fueron:

1. la necesidad de hacer del aprendizaje profesional el corazón de los asuntos escolares;

2. la necesidad de que el DPD se centre en los resultados de los alumnos;

3. la necesidad del desarrollo de contenidos pedagógicos profundos y conocimiento de la evaluación cuando los resultados se centran en áreas académicas; $y$

4. la necesidad de construir entornos de aprendizaje profesional que sean coherentes con las formas en las que se aprende.

En Australia, existen diferentes vías de promover el DPD del profesorado y de los formadores de docentes, incluyendo talleres de día y de medio día, módulos de 12 a 15 horas de duración (que en algunos casos pueden convalidarse por créditos universitarios oficiales, cuando los profesores realizan cursos completos de Máster y Doctorado), tutorías y supervisión, proyectos de investigación-acción y muchas otras variantes sobre estos temas. Gran parte del DPD en Australia aún se imparte en formatos que implican la transmisión unidireccional de conocimientos entre el emisor 
(proveedor de DPD) y el receptor (el profesor que cursa el programa de DPD). Estos programas fomentan poca participación creativa de los profesores implicados y por lo tanto resultan más DPD impartido "hacia" los profesores que DPD llevado a cabo "por" o "con" los profesores. En esta versión común del DPD, estos tres elementos (el proveedor de DPD, el profesor, y el lugar de trabajo educativo) a menudo operan con total independencia unos de otros. Este enfoque de DPD puede llevar a un aumento de la información o del conocimiento que el profesor posee, pero rara vez lleva a cambios reales en la práctica. En la mayoría de los casos, se produce poca o ninguna transferencia del conocimiento o de la práctica de los participantes en DPD hacia sus compañeros.

A pesar de estas críticas, dicho enfoque es capaz de encender una chispa que, si se sigue rápidamente, puede conducir a una exploración más profunda de un tema, enfoque o concepto. Si un grupo de personas de un mismo lugar de trabajo educativo asiste a sesiones de DPD de este tipo, algunas veces pueden continuar trabajando juntos y explorando su aprendizaje al volver a su escuela. Sin embargo, con la presión del trabajo y sin el apoyo de los directores educativos para hacerlo, en general después de aproximadamente una semana, la práctica de los participantes no demuestra que hayan participado en DPD, aunque hayan ganado en términos de conocimiento. Lo que suele faltar en este enfoque es la oportunidad de una interacción dinámica entre los proveedores del DPD, los participantes y las escuelas.

Un enfoque de DPD más dinámico es aquel donde los tres elementos principales - el proveedor del DPD, los participantes y los lugares de trabajo educativos - están involucrados en la negociación y el diseño colaborativo del propio DPD, satisfaciendo necesidades identificadas con anterioridad o objetivos específicos. Mediante la interacción dinámica entre los tres actores clave del DPD, todas las partes pueden involucrarse activamente en las diferentes etapas del proceso de DPD. La expectativa de este enfoque es que haya espacio para el cambio continuo y para la adaptación de actividades a lo largo del proceso de DPD, como resultado de la interacción entre los tres actores clave antes citados. En el contexto australiano actual, están presentes tanto el enfoque didáctico-tradicional de DPD como el segundo enfoque más interactivo y dinámico.

\section{¿Cuánto DPD es Necesario?}

En cuanto a la frecuencia de DPD, en la mayoría de los estados en Australia actualmente se especifica un número mínimo de horas de DPD que los profesores deben realizar en un período de tiempo determinado. No obstante, dicha política es reciente en ciertos casos. A diferencia de los órganos legislativos que rigen otras profesiones, los departamentos de educación y los organismos de acreditación en Australia no han requerido DPD en el pasado con consistencia o de modo particularmente robusto. Cole (2012) sostiene que un DPD obligatorio "tiende a reforzar la frecuente falsa percepción de que existe un fuerte vínculo entre el aprendizaje profesional, la mejora en la práctica docente y la mejora de toda la escuela" ["tends to reinforce the often false perception that there is a strong link between professional learning and improved teaching practice and whole school 
improvement"] (p.5). Existe también la posibilidad de que convertir el DPD en obligatorio convierta esta actividad en un mero trámite para los profesores, llevándoles a completar las horas requeridas de la manera más superficial posible para simplemente conservar su acreditación docente. Pese a ello, desde noviembre de 2008, se ha obligado a los profesores de Victoria a declarar que han llevado a cabo una "cantidad definida y determinada de actividades de desarrollo profesional al solicitar la renovación del acreditación docente" (véase FAQs, vit.vic.edu.au). La "cantidad definida y determinada" se traduce en un mínimo de 20 horas de DPD relacionadas con los Estándares de los Profesionales Australianos anuales para profesores. Este reglamento establece que "las actividades de desarrollo profesional deben actualizar los conocimientos de pedagogía, en su contenido y/o en su práctica" (véase FAQs, vit.vic.edu.au)". Otros estados y territorios tienen requisitos similares. Sin embargo, muchos profesores superan las 20 horas requeridas para tener derecho a la renovación del acreditación docente, ya que las categorías y tipos de DPD pueden estar compuestas por actividades formales e informales en muchas áreas.

Un estudio encargado por el Departamento de Educación, Empleo y Relaciones Laborales de Australia (DEEWR, 2011), sin embargo, encontró que los profesores de escuelas primarias en Australia habían participado una media de 9 días en actividades de DPD (organizadas tanto formal como informalmente) durante el año anterior, mientras que los profesores de secundaria participaron 7,6 días. Más de la mitad de los profesores que participaron en actividades de DPD eran profesores especialistas en el área de lectoescritura, aritmética y necesidades especiales, con una mayor proporción de participantes en lectoescritura. La participación más baja en áreas especializadas de DPD fue encontrada en los profesores de Informática. Para los profesores de secundaria de las siete áreas de especialización consideradas (Biología, Física, Química, Ciencia General, Geografía, Historia, e Informática) menos de la mitad de los profesores habían participado en alguna actividad de DPD reconocida. Los mayores niveles de participación en DPD en los docentes de secundaria encuestados se dieron en Inglés y en Idiomas distintos al Inglés (LOTE).

Sin embargo, participar en mayor cantidad de actividades de DPD no significa que éste vaya a ser necesariamente eficaz. Dar rigor científico a las discusiones sobre el DPD es clave para determinar qué constituye DPD efectivo. Este es el foco de gran cantidad de investigación en Australia en estos momentos.

\section{¿Qué es la Eficacia del DPD y Cómo se Mide?}

Hace casi 20 años, Mackenzie (1997) propuso que el DPD sólo puede ser considerado efectivo si conlleva un cambio en la práctica, y por ende, mejora los resultados de los alumnos. Sin embargo, en aquel momento Mackenzie argumentó que la mayoría del DPD parecía conducir a cambios en el conocimiento de los profesores, pero no a cambios en la práctica. En un estudio posterior realizado por el Departamento de Educación, Empleo y Relaciones Laborales (DEEWR, 2011) en Australia, se encontró de que la mayoría de profesores encuestados describió el DPD 
en que participaron como valioso en términos de su propio conocimiento y desarrollo de habilidades. Este resultado que sugiere que "la transferencia de conocimiento" sigue siendo el foco de muchos de los profesores al participar en DPD. Los profesores también afirmaron que el DPD había incrementado su eficacia al promover el aprendizaje de los alumnos en mayor o menor grado. No obstante, es necesario tener en cuenta que los datos se recogieron mediante auto-informes. Los profesores expresaron que las áreas específicas en que necesitaban mayor ayuda eran: metodología para la evaluación del aprendizaje de los alumnos; métodos para involucrar a los alumnos en las materias; conocimiento de los contenidos; desarrollo de materiales didácticos relevantes; planificación de los objetivos de aprendizaje para los alumnos; y la ampliación de la gama de asignaturas que son capaces de enseñar (DEEWR, 2011).

Las áreas identificadas por los profesores como focos que requieren atención en DPD señalan las necesidades percibidas por parte de los profesores de aprender activamente nuevas habilidades y estrategias de enseñanza. Se sugiere aquí que el aprendizaje profesional debe adquirir una consideración central si el cambio en la práctica docente y la mejora del aprendizaje de los alumnos son el resultado deseado del DPD. El aprendizaje del profesor, según Ofter y Pedder (2011), debe centrarse en una interacción recíproca entre el profesor, la escuela y la actividad de aprendizaje en la que el profesor esté involucrado. Esto es congruente con el estudio realizado por Cole (2012), quien llegó a la conclusión de que en el contexto de Australia, un DPD efectivo necesita ser "contextualizado en o directamente relacionado con el trabajo de enseñar, arraigado en los contenidos de enseñanza, organizado en torno a la resolución colaborativa de problemas, e integrado en un proceso de cambio integral" ["embedded in or directly related to the work of teaching, grounded in the content of teaching, organised around collaborative problem solving, and integrated into a comprehensive change process"] (p.7). Estos resultados se alinean con el enfoque dinámico e interactivo descrito anteriormente, donde todas las partes -el proveedor del DPD, los participantes y el lugar de trabajo educativo - están involucradas de forma recíproca, interactiva y dinámica en todas las etapas del programa de DPD.

Respecto al tiempo transcurrido entre las actividades de DPD y el momento en que pueden observarse cambios en las prácticas de los profesores, Cole (2012) encontró que:

"entre el momento en que el aprendizaje profesional se produce, la práctica docente cambia y el aprendizaje de los alumnos mejora, la multiplicidad de influencias que puedan contribuir a que profesor realice los cambios en su práctica hacen que la investigación sobre las "mejores prácticas" de aprendizaje profesional sea problemática en esta etapa" [between when professional learning occurs, teacher practice changes and student learning improves, and the multiplicity of influences on a teacher that could contribute to changes in their practice, makes research into "best practice" professional learning fairly problematic at this stage] (p.8). 
Cole (2012) sugiere que el DPD más eficaz en términos de producir cambios en la práctica docente y mejoras en el aprendizaje de los alumnos se inscribe en la práctica rutinaria de la escuela. En el DPD más eficaz, profesores "expertos" trabajan con otros profesores en sus aulas o grupos de profesores trabajan conjuntamente y aprenden de forma colaborativa. Cole también encontró que el DPD efectivo:

1. se centra en el dominio de estrategias y técnicas de enseñanza, incluyendo estrategias y técnicas de micro-enseñanza;

2. es un esfuerzo de toda la escuela diseñado para producir la mejora de las prácticas de enseñanza con base en datos;

3. implica que los profesores desarrollen planes individuales de aprendizaje profesional y los comparan con otros profesores, de forma que los profesores compartan enfoques similares de DPD y se apoyen mutuamente. Estos planes deben ser revisados y renovados regularmente.

Existen diferentes factores que tienden a mermar la eficacia del DPD. Estos factores se centran principalmente en torno a:

1. la reticencia de los profesores a tutorizar o supervisar a sus compañeros;

2. la reticencia de los profesores a pedir ayuda o apoyo por parte de sus compañeros;

3. la falta de tiempo para participar en las actividades de DPD; y

4. la negativa a admitir la importancia del DPD y del apoyo colegial (Cole, 2012).

Lo que se necesita, según Cole (2012), es que se establezcan culturas de aprendizaje profesional en todas las escuelas, con profesores que asuman la responsabilidad en dichas culturas. Esto facilitaría que los profesores altamente experimentados apoyen $y$ supervisen formalmente a sus colegas menos experimentados. Una conclusión final y reveladora de este estudio australiano es que

"En un contexto escolar donde los profesores no son colaborativos y no están acostumbrados a compartir ideas y experimentar con su práctica docente, es poco probable que los programas de aprendizaje profesional sean eficaces, incluso aquellos con un contenido sólido y estrategias de formación potentes" In a school context where teachers are not collaborative and used to sharing ideas and experimenting with their teaching practice, even professional learning programs with solid content and powerful training strategies, are unlikely to be effective] (Cole, 2012, p.14).

En otro estudio realizado en Australia, Ingvarson, Meier y Beavis (2005) revisaron cuatro programas de DPD específicamente diseñados para mejorar la calidad del profesorado. Los autores entrevistaron a los profesores al menos tres meses después de su participación en las actividades del DPD. En este estudio, se identificaron cinco características principales del DPD efectivo: centrado en el 
contenido que se enseña, se ofrece seguimiento a los profesores, su aprendizaje es activo, se ofrece retroalimentación a los profesores, y el trabajo de los alumnos se analiza colaborativamente. El impacto de los programas se midió en términos del conocimiento del profesor, su práctica, los resultados del aprendizaje de los alumnos, y la eficacia del profesor, aunque los datos se basaron en auto-informes por parte de los profesores. No hubo discusión sobre el cambio en las prácticas.

Cole (2012), en el estudio australiano del DPD, afirma que en los estudios previos llevados a cabo en Australia por Corcoran (1995), Ingvarson (2003) y Supvitz y Turner (2000):

"revelan que el aprendizaje profesional generalmente consiste en actividades desenfocadas, fragmentadas, de baja intensidad, como si se impartieran en talleres a corto plazo con poco o ningún seguimiento; como consecuencia, la capacidad de la profesión para comprometer a la mayoría de sus miembros en modos aprendizaje profesional más eficaces de forma duradera ha sido débil" [reveal that professional learning generally consists of unfocused, fragmented, low-intensity activities, such as short-term workshops with little or no followup and consequently that the capacity of the profession to engage most of its members in effective modes of professional learning over the long term has been weak] (p.5).

En respuesta a la pregunta de qué constituye un DPD eficaz, este hallazgo es decepcionante y es parte del desafío al que nos enfrentamos para conducir al DPD a una nueva era. Ello nos lleva a plantearnos la siguiente pregunta: ¿Qué se necesita para cambiar las prácticas y para mejorar los resultados de los alumnos mediante DPD en la época actual? En la era de la supercomplejidad, donde el futuro es desconocido e incierto, en el que coexisten valores contrapuestos, con presiones y agendas a las que los profesores necesitan abordar y resolver, el DPD está obligado a facilitar y fortalecer ciertas habilidades clave y atributos en los profesores, que les prepare para enseñar en esta era. El DPD tendrá que contener un elemento importante de reflexión crítica, así como potencial para ayudar a los profesores a desaprender, cambiar y re-aprender habilidades de enseñanza y enfoques que ya no funcionen o no sean apropiados. Además, el DPD tendrá que animar a los profesores a confrontar y cuestionar su comprensión de lo que es ser un profesor y de lo que los estudiantes necesitan para enfrentarse al presente y al futuro. Es más probable que el tipo de reflexión crítica que esto requiere se dé cuando se involucran activamente a los proveedores del DPD, a los profesores y a las escuelas en una interacción dinámica. El DPD basado en la escuela descrito por Cole (2012) será probablemente más eficaz cuando los profesores y sus mentores trabajen de manera rutinaria y continua en las escuelas. Dicho enfoque refleja el modelo interactivo dinámico del DPD, en lugar del modelo de transmisión didáctica unidireccional.

\section{DPD para la Supercomplejidad}

En una era de supercomplejidad, cualquier DPD que se centre en habilidades y conocimientos sólo puede ser útil realmente para el pasado o, como mucho, para el 
presente inmediato, ya que no sabemos con precisión qué habilidades serán más necesarias en las escuelas desconocidas del futuro. Más bien, necesitamos un DPD que se centre en el desarrollo de las "cualidades y disposiciones humanas" [human qualities and dispositions] (Barnett, 2012, p.65). Esto implica que los profesores tendrán que analizar reflexiva y radicalmente el ser y esencia de la enseñanza y el aprendizaje, así como las escuelas y sus propósitos. Esto requerirá un cambio en la manera en que conceptualizamos el aprendizaje y los significados que le atribuimos. También necesitamos repensar el ser mismo del aprendizaje y lo que significa ser un aprendiz en la época actual y en el futuro. El DPD tendrá que ayudar a los maestros a desarrollar disposiciones y atributos que les preparen para convivir con la incertidumbre, el cambio constante, los valores y demandas múltiples y a menudo en conflicto, así como para aceptar que su propio conocimiento es provisional, siempre abierto a la reinterpretación y el cuestionamiento, y con marcos de referencia diferentes y cambiantes.

Este enfoque contrasta con la mentalidad del "sabio en el escenario" y favorece la mentalidad de "guía al lado", reforzando la importancia del co-aprendizaje y enfatizando que los profesores y los estudiantes han de desempeñar un rol conjunto en la creación de significados y de conocimientos. En un mundo supercomplejo, los profesores en todos los sectores de la educación tendrán que introducir lo "extraño" en las experiencias propias y en las de sus alumnos (Barnett, 2015). Para Barnett, esto implica que los seres humanos tendremos que adaptarnos a situaciones complicadas en las que ignoremos el futuro, o con un conocimiento provisional en el mejor de los casos, por lo que tendremos que estar cómodos asumiendo riesgos y aceptando el cambio y la inseguridad como la norma, en lugar de algo que hay que resolver o inamovible. Con base en estas ideas, Barnett (2015) se refiere a la enseñanza para un futuro desconocido como:

“... la producción de las capacidades, cualidades y disposiciones humanas para la asimilación personal y la creación de extrañeza. Tal concepción de la enseñanza busca una ruptura fundamental con las relaciones pedagógicas convencionales y se orienta hacia planes de estudio que presenten espacios incómodos para los alumnos. Por medio de estos espacios, los alumnos tomarán conciencia de sus propias capacidades para asimilar e incluso producir extrañeza" [... the production of human capacities, qualities and dispositions for the personal assimilation and creation of strangeness. Such a conception of teaching looks to a fundamental break with conventional pedagogical relationships and looks to curricula that present awkward spaces to and for the students. Through such spaces, the learner will realize for themselves their capacities for assimilating and even producing strangeness] (p.94).

Esta ruptura fundamental con las relaciones pedagógicas convencionales también tendrá que reflejarse en la pedagogía del DPD. El tipo de pedagogía requerida en el mundo de la supercomplejidad tendrá que ser eminentemente abierta 
y flexible para ayudar a los profesores a ser adaptables, y sobre todo a estar cómodos a la hora de correr riesgos con su propio aprendizaje, de manera que también aprendan a estimular a sus alumnos a arriesgarse como base del aprendizaje. En términos prácticos, esto conllevaría implicar a los profesores en actividades de DPD que requieran habilidades diferentes a las que suelen utilizar normalmente. Esto puede lograrse haciendo que los profesores se enfrenten a conceptos que desafían sus prácticas o conocimientos existentes, lo que les llevará a ampliar su pensamiento más allá de su zona de confort actual y tradicional. Este enfoque de DPD requiere que los profesores, a través de actividades de apoyo en talleres y sesiones de microenseñanza, experimenten con nuevos métodos de enseñanza, nuevos enfoques de gestión en el aula, y formas innovadoras y creativas para alcanzar exitosos resultados de aprendizaje.

Los enfoques de DPD que reflejan este punto de vista incluyen aquellos que invitan a profesores a involucrarse en proyectos de investigación, debatiendo con otros profesores-investigadores e investigadores profesionales hasta encontrar soluciones a problemas pedagógicos. Inicialmente, los problemas pueden ser confusos y casi inabordables, permitiendo de esta forma que los participantes se involucren en la ideación de soluciones, siempre con un entendimiento compartido sobre la meta hacia dónde se dirige el grupo. Los participantes son vistos como contribuyentes al aprendizaje de todos, en vez de entenderse que los profesores necesitan que alguien les ayude a desarrollarse.

Al diseñar actividades de DPD eficaces para los profesores, es necesario que los participantes puedan llegar a resultados consensuados a través de una variedad de medios diferentes y del uso de diversidad de materiales y enfoques o estrategias. Esto exige sesiones de DPD abiertas, en las que la toma de riesgos se estimule e incluso ineludible, a fin de alcanzar los resultados esperados. Se incorporará el riesgo como resultado necesario para tener éxito. Por ejemplo, "¿Cuántos enfoques de enseñanza y aprendizaje diferentes puedes mostrar y describir para ayudar a tus alumnos a utilizar diversos géneros en su escritura?" Es posible que una sesión de DPD centrada en animar a los profesores a probar y crear este tipo de enfoques, así como a debatir conjuntamente como una comunidad de aprendices, anime a los profesores a utilizar dichos enfoques con sus alumnos en clase, especialmente si tienen algún compañero con quien puedan debatir y colaborar.

Una de las autoras de este artículo (Mackenzie) está implicada en un programa de este tipo. Dicho programa se describe a continuación como ejemplo de DPD que incluye los elementos que hemos señalado como necesarios en una era de supercomplejidad. El proyecto "Entendiendo y Apoyando los Proyectos de Jóvenes Escritores" está en marcha en Victoria desde el año 2015. Es este proyecto, un investigador universitario y dos consultores de la Autoridad Curricular y de Evaluación de Victoria han reunido a 14 maestros de educación infantil (7 de contextos preescolares y 7 de contextos escolares) para encontrar las mejores formas de a la escritura. Todos los participantes se ofrecieron como voluntarios para trabajar en colaboración y para probar los enfoques que ellos mismos sugerían, partiendo de sus propios contextos de enseñanza y aprendizaje, así como para compartir los 
resultados con el grupo. En este tipo de situación, los participantes suelen están nerviosos inicialmente, y se siente extraños e incómodos cuando se dan cuenta de que están generando conocimiento, más nerviosos que si solamente estuvieran recibiendo conocimiento. El investigador principal (Mackenzie) ha utilizado este método con éxito con profesores del estado australiano de Nueva Gales del Sur. Si bien el proceso se articula como investigación y sigue todas las pautas éticas de la investigación, uno de los principales resultados es el aprendizaje profesional de los docentes. Se utilizan muestras auténticas de escritura y dibujo de niños, algunas de ellas controvertidas, para estimular discusiones, debates y dilemas. Este enfoque contrasta directamente con otras formas didácticas de DPD, que dogmáticamente defienden enfoques prefijados de forma prescriptiva y cerrada.

Como hemos señalado aquí y en otros lugares (Ling y Mackenzie, 2001), el DPD puede adoptar muchas formas. La cuestión, sin embargo, es si de cualquiera de estas formas frecuentes hoy día en Australia y en otros lugares se están formando profesores para un contexto de enseñanza incognoscible y supercomplejo. Cole (2012) sostiene que, debido al rápido cambio en la enseñanza y el aprendizaje en Australia, las "nuevas y mejores formas de aprendizaje profesional son prometedoras pero su eficacia no está todavía probada " [new and improved forms of professional learning are still at the "looks highly promising but not proven] (p.7). Recientemente se han producido cambios positivos en el área del DPD en Australia, , aunque todavía hay margen para profundizar en su mejora.

Con base en esta discusión sobre las características del DPD eficaz en la era actual, proponemos una forma de DPD que comience a abordar los desafíos de la supercomplejidad y la extrañeza que implica el aprendizaje de los profesores. Este enfoque dinámico, interactivo y colaborativo, donde los proveedores del DPD, los profesores y los lugares de trabajo participan activamente en todas las etapas del programa de DPD, donde las actividades están insertas en el aula, en lugar de ser un añadido "extra", proporcionan el marco para un DPD eficaz para un futuro desconocido. Este enfoque conduce potencialmente a la energía, el impulso y el cambio de todos los agentes involucrados: los profesores; la escuela o escuela infantil; el sistema; y los proveedores del desarrollo profesional.

El proyecto "Entendiendo y Apoyando los Proyectos de Jóvenes Escritores" descrito anteriormente está adoptando este enfoque interactivo, colaborativo y contextualizado, mostrando gran potencial para el cambio de todos los elementos. Dicho cambio está siendo facilitado de forma interactiva y colaborativa por el investigador principal (el proveedor), los consultores del DPD (el sistema), y los educadores (los profesores participantes) que proceden de 14 entornos educativos diferentes. Cada miembro tiene el potencial de crecer como resultado de su participación en el proyecto. Con el apoyo adecuado en el lugar de trabajo, la expectativa es que cada profesor propiciará el cambio dentro de su propio centro, que los directivos de la VCAA iniciarán también el cambio a través de su propio sistema, y que el investigador principal tratará de propulsar el cambio de manera más amplia a 
través de publicaciones y la difusión de los resultados y conclusiones del proyecto. Pese a que dicho proyecto está aún en curso, ya podemos comunicar algunas de las primeras reacciones de algunos participantes de cada uno de los tres grupos de agentes.

Como investigador, este tipo de enfoque hacia el DPD mucho más gratificante y estimulante para mi que el DPD directo y tradicional. También es mucho más exigente. Cuando doy presentaciones públicas, incluso si el tema ha sido determinado por el grupo, puedo controlar mi lenguaje corporal e involucrar a la audiencia en buena medida, pero no obtengo ninguna respuesta real hasta después del evento. No sé si realmente he conectado con los participantes, o qué se produce después de mi exposición. En proyectos como el descrito aquí, empiezo con un marco negociado pero respondo reflexivamente al grupo. Yo soy parte del grupo, más que un "sabio en un escenario", y la dirección que siguen los debates es determinada por el grupo. (participante 1 - Proveedor de DPD)

Dado que nos reunimos muy asiduamente, las relaciones se van estableciendo y las contribuciones de los miembros del grupo van cambiando a medida que nuestra confianza aumenta. El análisis de los ejemplos compartidos por los alumnos se suma a la autenticidad y a la dirección de nuestras discusiones. (participante 2 - Consultor del DPD)

Este enfoque es colaborativo e interactivo. Generalmente continúo reflexionando sobre las discusiones durante días después de cada sesión. Estas reflexiones conforman el punto de partida para la sesión siguiente. (participante 3 - Profesor participante)

Como se refleja en los comentarios de los participantes, los elementos clave que hacen de ésta una experiencia de DPD diferente y valiosa es su base en la interacción entre los participantes, su carácter colaborativo, el ser una experiencia coconstruida por ellos mismos y por el proveedor del DPD, el ser relevante para su trabajo y sus necesidades, y el hecho de hacerles reflexionar incluso una vez las sesiones han concluido. No es una transmisión didáctica y unidireccional de información del proveedor del DPD hacia los participantes. En el DPD descrito anteriormente, los participantes trabajaban con el proveedor para dar forma a las actividades y tenían un papel activo. Dicho enfoque hacia el DPD, por tanto, tiene un efecto más penetrante en los participantes, pues le permite integrar los conceptos que han abordado en las sesiones de DPD en su trabajo y reflexionar sobre ellos. 
Tabla 1. Resultados preliminares de "Entendiendo y Apoyando los Proyectos de Jóvenes Escritores"

\begin{tabular}{|c|c|}
\hline Resultados provisionales & Evidencia \\
\hline $\begin{array}{l}\text { - Aumento de la conciencia hacia un } \\
\text { contexto más amplio de lecto-escritura y } \\
\text { formas de apoyo a los niños en ese } \\
\text { contexto } \\
\text { - El aumento de la capacidad de ser } \\
\text { reflexivo }\end{array}$ & $\begin{array}{l}\text { - Respuestas reflexivas de los participantes a } \\
\text { lecturas, provocaciones y escenarios } \\
\text { - El análisis compartido de los participantes } \\
\text { sobre las muestras de escritura/dibujo en los } \\
\text { contextos de la escuela y de la primera infancia } \\
\text { muestran cambios en el pensamiento y en el } \\
\text { discurso }\end{array}$ \\
\hline $\begin{array}{l}\text { - Aumento de toma de riesgos y confianza } \\
\text { por parte de los participantes } \\
\text { El aumento de la auto-eficacia en lo } \\
\text { relativa a la enseñanza de los jóvenes } \\
\text { escritores }\end{array}$ & $\begin{array}{l}\text { - Disposición de los participantes para compartir } \\
\text { abierta y confiadamente y para hacer preguntas } \\
\text { - Muestras de trabajo compartido entre los } \\
\text { participantes muestran un cambio en lo que se } \\
\text { promueve y se valora }\end{array}$ \\
\hline $\begin{array}{l}\text { - Aumento de la voluntad de compartir la } \\
\text { evidencia del trabajo con los niños } \\
\text { - Aumento de la confianza en el análisis } \\
\text { de las muestras de trabajo de los niños } \\
\text { - El aumento de la preparación para } \\
\text { compartir los retos y sus posibles } \\
\text { soluciones }\end{array}$ & $\begin{array}{l}\text { - El número y la variedad de las muestras que se } \\
\text { comparten, la disposición de los participantes a } \\
\text { comentar y discutir. } \\
\text { - Comentarios anecdóticos unidos a las muestras } \\
\text { de trabajo de los niños que han sido provistas a } \\
\text { los investigadores por parte de los participantes } \\
\text { revelan un análisis más profundo y una mayor } \\
\text { comprensión }\end{array}$ \\
\hline $\begin{array}{l}\text { El desarrollo de un vocabulario común para } \\
\text { la discusión de los jóvenes escritores y la } \\
\text { enseñanza y el aprendizaje de los procesos } \\
\text { que los respaldan }\end{array}$ & $\begin{array}{l}\text { Si bien los participantes tenían ideas similares al } \\
\text { comienzo, sus discusiones reflejan que en } \\
\text { ocasiones sus ideas también diferían. Los } \\
\text { participantes de las escuelas compartían un } \\
\text { lenguaje común, y los participantes de centros de } \\
\text { cuidado infantil temprano otro, por lo que hubo } \\
\text { diferentes interpretaciones entre dichos grupos. Se } \\
\text { pasó tiempo 'bromeando' sobre los términos y el } \\
\text { glosario desarrollado y compartido. Esto ayudó a } \\
\text { redefinir los debates y dar lugar a significados y a } \\
\text { un discurso compartido. }\end{array}$ \\
\hline Mayor interés en aprender más & $\begin{array}{l}\text { Los participantes demostraron interés en leer más } \\
\text { acerca de los temas compartidos durante las } \\
\text { sesiones grupales y en discutirlos con sus } \\
\text { compañeros. }\end{array}$ \\
\hline
\end{tabular}

El enfoque del DPD descrito anteriormente no se basa predominante o exclusivamente en habilidades concretas, sino que ayuda a los profesores a prepararse para la supercomplejidad mediante el desarrollo de la reflexión crítica, estrategias de toma de riesgos, creatividad, tenacidad e ingenio respecto al aprendizaje y la enseñanza de áreas extrañas y difíciles, con las que tanto profesores como estudiantes pueden encontrarse. Para desarrollar estas disposiciones a través del DPD, los profesores tienen que desafiar las creencias y puntos de vista existentes, y ser lo (C) Psy, Soc, \& Educ, 2015. Vol. 7(3) 
suficientemente valientes como para resistirse a los métodos de enseñanza que no tengan en cuenta su punto de vista o el de sus estudiantes. Aquellas actividades que desafíen a los profesores, evaluando críticamente los currículos y planes de estudio, documentos, métodos de enseñanza, políticas educativas, prácticas de evaluación, y la cultura docente existente, conducirán finalmente a una voluntad de cambio y a emprender acciones que lo promuevan. El proyecto "Entendiendo y Apoyando los Proyectos de Jóvenes Escritores" es un ejemplo de programa de DPD que implica el desarrollo de las características aquí discutidas. Aunque el programa todavía estaba en curso cuando este artículo se envió a la imprenta, la Tabla 1 presenta algunos resultados preliminares de las encuestas, reflexiones y entrevistas que llevamos a cabo.

De este modo, la tarea de los proveedores de DPD es ofrecer a los profesores oportunidades para reflexionar sobre su propio trabajo y el de los demás, a la par que presentarles dilemas contextualizados que se relacionen con su propia realidad en el aula y que a su vez reflejen parte de la confusión desordenada a la que se enfrentan al tratar de hacer frente a estos dilemas. Si los profesores comparten su propia situación, confusiones y dilemas, las discusiones en las sesiones de DPD probablemente serán mucho más auténticas y significativas para ellos que otras actividades sin relevancia para el trabajo de un profesor. Los profesores han de ser el centro del DPD.

\section{Los Profesores como Centro del DPD}

Al examinar el DPD efectivo, Cole (2004) observó que los profesores tienen visiones extremadamente reducidas sobre lo que constituye el DPD, entendiendo que es algo que los profesores hacen fuera de la escuela. Cole sugiere que el término "desarrollo profesional" debería sustituirse por el de "aprendizaje profesional", ya que hace hincapié en el aprendizaje docente y por lo tanto coloca al profesor en el centro de la acción. Sin embargo, para conseguir que los profesores experimenten y sean capaces de aceptar este aprendizaje profesional, a veces es necesario plantearles algo que les cause alguna dificultad. Ello provoca que las áreas difíciles y las situaciones desconocidas lleven a los profesores a cuestionar o refutar algunas de sus prácticas habituales. Los profesores necesitan oportunidades y motivación para desaprender y volver a aprender a través del aprendizaje profesional. Lograr que el DPD fortalezca a los profesores y fomente su sentido de autoeficacia requiere realizar actividades como las llevadas a cabo en ciertos estudios en Australia.

Black (1999) describe algunas actividades de DPD que ella pidió a los profesores que aportaran un dibujo de sí mismos como docentes y que escribieran una descripción del dibujo. Posteriormente, estas producciones se convirtieron en el foco del debate y la discusión entre compañeros. Black también pidió a los profesores que crearan una metáfora para su dibujo, argumentando que esto fomenta la autoexploración de los "vínculos entre el conocimiento práctico y la propia práctica docente" (p.9). Black también utilizó la escritura de historias y el uso de revistas, combinándolas con reflexiones como piezas narrativas.

En otras actividades de DPD llevadas a cabo por los autores de este artículo, se pidió a los profesores que pensaran en situaciones en las que se hubieran sentido 
fortalecidos y después en otras en las que se hubieran sentido con menos poder, prosiguiendo con una discusión sobre las características específicas de dichas situaciones. Esta actividad puede promover ricos debates sobre cómo los profesores pueden tomar el control de situaciones en que se sienten con menos poder, y qué estrategias podrían utilizar para convertirlas en situaciones de fortalecimiento. Esto es especialmente importante en el DPD para los posibles directores, ya que les ayuda a saber cómo crear situaciones de fortaleza para los profesores de su centro y para los estudiantes. A través de este tipo de experiencias, los profesores pueden darse cuenta también de que ésta es la clase de aprendizaje que sus propios alumnos necesitan para hacer frente a un mundo de supercomplejidad. De esta manera, el objetivo no es sólo que los profesores se involucren en un nuevo aprendizaje y nuevas formas de ser-enel-mundo, sino que sus alumnos participen de estos procesos. Así, el DPD abordaría de una forma más directa la cuestión clave de cambiar los resultados de aprendizaje de los alumnos de forma positiva.

\section{Conclusión}

En Australia, los directores escolares son quienes deciden mayormente cómo se invierten los fondos para el DPD. En efecto, los equipos directivos de las escuelas tiene gran influencia sobre qué DPD se ofrece a los profesores. El DPD es fundamental en vistas a conseguir cambios o resultados positivos en los profesores y en los alumnos. Esto requerirá directores que puedan correr riesgos y que desafíen constantemente tanto sus propias acciones como su noción de lo significa ser un líder-en-el-mundo de hoy día. Es imprescindible que los directores, profesores y aquellos que diseñan el DPD desarrollen capacidades que les permitan aceptar la supercomplejidad y lo desconocido. Sin embargo, es en el nivel sistémico educativo en donde se necesita hacer los cambios de forma masiva.

En Australia, como en muchos otros países en la actualidad, se han convertido en la norma el hecho de comparar el rendimiento de los estudiantes mediante pruebas estandarizadas, o comparar el rendimiento de las escuelas y de las universidades en tablas comparativas. El DPD ofrecido por los departamentos de educación se centra casi inevitablemente en políticas específicas y agendas que influyen en las decisiones curriculares de los profesores, en enfoques de enseñanza y resultados de aprendizaje, en las prácticas de evaluación y en programas específicos, así como en materiales curriculares prescritos para ser utilizados en las escuelas. Las sesiones de este tipo suelen ser de medio día o de un día de duración. Se espera que los participantes regresen a sus escuelas y difundan las ideas y estrategias aprendidas entre sus colegas. Los resultados que se esperan de estos programas de DPD están altamente prefijados y los materiales son frecuentemente prescriptivos en cuanto cómo los profesores y los alumnos han de utilizarlos. Si se espera que los profesores desarrollen disposiciones críticas y reflexivas que les permitan lidiar con la incertidumbre y con interpretaciones diversas, y que les permitan incluso desafiar sus propias identidades y roles, como hemos defendido más arriba, el tipo de programas 
de DPD ofrecidos típicamente por las universidades y el gobierno y sus agencias están abocados al fracaso. Incluso peor, estos programas tienden a fomentar la conformidad de los profesores, el cumplimiento y la rendición de cuentas.

Sin embargo, hay otros tipos de DPD ofrecidos a través de universidades o de otros proveedores privados e independientes que, en algunos de los casos (aunque ciertamente no en todos), desafían a los profesores para que prueben nuevos enfoques de enseñanza, proporcionado un contexto colegial de apoyo donde desarrollar estas capacidades. Hemos hecho hincapié en la necesidad de que el DPD permita la interactividad y las relaciones dinámicas entre todos los agentes involucrados. El proyecto "Entendiendo y Apoyando los Proyectos de Jóvenes Escritores" ha sido un ejemplo del DPD que permite a los profesores desafiar su identidad, así como sus conocimientos, prácticas y creencias. Algo similar sucede con el enfoque utilizado por Black (1999), donde los profesores utilizan el dibujo, la metáfora, el relato de historias y la narración, así como revistas de reflexión, desafiando a los profesores a repensar su identidad y su papel como maestros. Cole (2012) también ha llegado a la conclusión de que las actividades de DPD han de contextualizarse en las escuelas, como parte de su funcionamiento diario. Especialmente eficaz son aquellos contextos donde los profesores-mentores trabajan las aulas con otros profesores. El DPD contextualizado en la escuela permite facilitar el cambio de toda la escuela, a diferencia del modelo de DPD unidireccional, donde no hay transferencia o intercambio de los conocimientos entre los participantes. En sesiones de DPD colaborativo, colegial y de apoyo, junto con el seguimiento en las escuelas y en sesiones posteriores, los profesores discuten, debaten y se cuestionan a sí mismos, el currículo, la política, la evaluación, los métodos de enseñanza y aprendizaje, y la esencia misma de lo que significa ser un profesor en un mundo frágil e incierto y con un futuro desconocido.

Es preciso dar a conocer los resultados y los beneficios positivos de estos enfoques del PDP, en una nueva era para el aprendizaje de los profesores. Del mismo modo, es importante comenzamos a hacer incursiones en el cambio de la cultura del DPD en las escuelas, a fin de hacerlo valioso y apropiado en nuestro actual mundo súper-complejo.

\section{Referencias}

Australian Institute for teaching and School Leadership (2011). A background paper to inform the development of a national professional development framework for teachers and school leaders. Dr Helen Timperley. AITSL.

Barnett, R. (2000). University knowledge in an age of supercomplexity. Higher Education 40, 409-422.

Barnett, R. (2011). Realizing the University in an Age of Supercomplexity. Ballmoor Bucks: The Society for research into Higher Education and OUP.

Barnett, R. (2012). Learning for an unknown future, Higher Education Research \& Development, 31(1), 65-77.

Barnett, R. (2015). Thinking and Rethinking the University in The Selected Works of Ronald Barnett (e-book). Routledge Oxon and NY. 
Black, A. L. (1999). Empowering Teachers: Using teaching Images to Understand Self. Creche and Kindergarten Annual Early Childhood Conference, Brisbane, Australia (unpublished).

Cole, P. (2004). Professional development: A great way to avoid change IARTV. Melbourne, Seminar Series No 140.

Cole, P. (2012). Linking effective professional learning with effective teaching practice. AITSL.

Ingvarson, L., Meiers, M., \& Beavis, A. (2005). Factors affecting the impact of professional development programs on teachers' knowledge, practice, student outcomes and efficacy. Education Policy Analysis Archives, 13(10), 1-28.

Lauer, P. A., Christopher, D. E., Firpo-Triplett, R., \& Buchting, F. (2014). The impact of short-term professional development on participant outcomes: a review of the literature, Professional Development in Education, 40(2), 207-227.

Ling, L. M., \& Mackenzie, N. (2001). The professional development of teachers in Australia. European Journal of Teacher Education, 24(2), 87-89.

Mackenzie, N. (1997). Professional Development: A Qualitative Case Study. Unpublished Masters thesis, La Trobe University, Albury-Wodonga Campus, Graduate School of Education.

Opfer, V., \& Pedder, D. (2011). Conceptualizing teacher professional learning. Review of Educational Research, 81(3), 376-407.

Possner, D. (2002). Education for the $21^{\text {st }}$ century, Phi Delta Kappan, 84(4), 316-317.

Taylor, M., Yates, A., Meyer, L. H., \& Kinsella, P. (2011). Teacher professional leadership in support of teacher professional development. Teaching and Teacher Education, 27(1), 85-94. http://dx.doi.org/10.1016/j.tate.2010.07.005 (accessed 22/3/2015).

Victorian Institute of Teaching, http:/www.vit.edu.au/registration/i-want-to-renew-myregistration/pages/keeping-records-of-professional-development.aspx $\quad$ (accessed 23/5/2015).

Victorian Institute of Teaching, http:/www.vit.edu.au/registration/i-want-to-renew-myregistration/registration-process-faqs/pages/default.aspx (accessed 23/5/2015).

Weldon, P., Rowley, G., \& McKenzie, P. (2011). Profiles of teachers in selected Curriculum Areas: Further Analyses of the Staff in Australia's Schools 2010 Survey, Canberra, Department of Education Employment and Workplace Relations. 